\title{
A Fuzzy-based Call Admission Control System for Wireless Cellular Networks
}

\author{
Leonard Barolli ${ }^{\dagger}$, Fatos Xhafa ${ }^{\ddagger}$, Arjan Durresi $^{\dagger \ddagger}$, Akio Koyama ${ }^{\ddagger \ddagger}$ \\ ${ }^{\dagger}$ Department of Information and Communication Engineering \\ Fukuoka Institute of Technology (FIT) \\ 3-30-1 Wajiro-Higashi, Higashi-ku, Fukuoka 811-0295, Japan \\ E-mail: barolli@ fit.ac.jp \\ ${ }_{\ddagger}^{\ddagger}$ Department of Languages and Informatics Systems \\ Polytechnic University of Catalonia \\ Jordi Girona 1-3, 08034 Barcelona, Spain \\ E-mail: fatos@1si.upc.edu \\ ${ }^{\ddagger}$ Department of Computer Science, Louisiana State University \\ Baton Rouge, LA 70803, USA \\ E-mail: durresi@csc.lsu.edu \\ $\ddagger \ddagger$ Department of Informatics, Yamagata University \\ 4-3-16 Jonan, Yonezawa 992-8510, Yamagata, Japan \\ E-mail: akoyama@eie.yz.yamagata-u.ac.jp
}

\begin{abstract}
The mobile cellular systems are expected to support multiple services with guaranteed Quality of Service $(Q o S)$. However, the ability of wireless systems to accommodate expected growth of traffic load and broadband services is limited by available radio frequency spectrum. Call Admission Control (CAC) is one of the resource management functions, which regulates network access to ensure QoS provisioning. However, the decision for CAC is very challenging issue due to user mobility, limited radio spectrum, and multimedia traffic characteristics. To deal with these problems, in this paper, we propose a fuzzy CAC system. We compare the performance of the proposed system with Shadow Cluster Concept (SCC). We evaluate by simulation the performance of the proposed system. The proposed system has a good behavior on deciding the number of accepted connections while keeping the QoS for serving connections.
\end{abstract}

\section{Introduction}

The future telecommunications networks (such as the third-generation and forth-generation wireless networks) aim to provide integrated services such as voice, data, and multimedia via inexpensive low-powered mobile computing devices over wireless infrastructures. As the demand for multimedia services over the air has been steadily in- creasing over the last few years, wireless multimedia networks have been a very active research area [1]. To support various integrated services with a certain Quality of Service (QoS) requirement in these wireless networks, resource provisioning is a major issue.

The QoS support for future wireless networks is a very important problem. To guarantee the QoS, Call Admission Control (CAC) is a good strategy. CAC is a provisioning strategy that limits the number of call connections into the networks in order to reduce the network congestion and call dropping. A good CAC scheme has to balance the call blocking and call dropping in order to provide the required QoS [1].

CAC is not a problem that is unique to wireless networks. It is applicable to almost every type of networks, but in cellular wireless networks due to users' mobility the CAC becomes much more complicated. While in wired networks the resources are reserved for the call at set-up time and are not changed after that, in cellular wireless networks when the mobile node moves from one cell to another one, the bandwidth must be requested in the new cell. During this process, the call may not be able to get a channel in the new cell to continue its service due to the limited resource in wireless networks, which will lead to the call dropping. Thus, the new and handoff calls have to be treated differently in terms of resource allocation. Since users are much more sensitive to call dropping than to call blocking, the handoff calls are assigned higher priority than new calls [2]. 
Future wireless networks are expected to support different broadband multimedia services with diverse QoS requirements. For more efficient utilization of the radio spectrum, the total area covered by a wireless network is divided into cells. Different traffic types can arrive at a cell. Traffic can also be handed over from other nearby cells when the mobile users cross cell boundaries. During the call setup phase, a cell must decide whether to admit a new (or a handed-over) call or not. This depends primarily on the availability of bandwidth to support the call. The amount of bandwidth allocated to a new call is based on various statistical properties of the call and on the number of calls already in session $[3,4,5]$. The call allowable delay and loss are specified by its QoS requirements. For example, voice traffic cannot be queued, because it cannot tolerate any delay, while data traffic is queue-able and a certain amount of delay can be acceptable.

$\mathrm{CAC}$ techniques are required to guarantee that all traffic types meet their QoS requirements. In order to improve the system performance at the call level (fairness in blocking), a CAC strategy may block additional calls even if there are enough resources for their service. CAC is based on the knowledge of the statistical characteristics of ongoing and arriving calls [2]. The decision to accept an additional call involves the calculation or estimation of the consequences of the call acceptance on blocking and delay of itself and other incoming calls [3].

Several schemes have recently been proposed for CAC in wireless cellular networks. One of the simplest CAC techniques is the Complete Sharing (CS) strategy. In CS, an arriving customer is served if there are enough free channels for its service. If the number of free channels is less than the channel requirements of the arriving customer, it is lost. This technique is easy to implement but it suffers from the fact that it is not fair to customers with large bandwidth requirements. The authors in [1] studied the performance of some widely known CAC protocols under more general (more accurate) assumptions and provided good approximations for the network performance. In [4], the authors proposed a model for heterogeneous multi-class environment that permits call transition between different classes. They also show that under some assumptions, the optimal policy has the shape of Multi-Priority Threshold Policy.

In [5], the authors proposes a CAC algorithm that can maintain the desired level of QoS, while the successful call completion rate is very high. In the proposed algorithm, the new call arrival rate is estimated continuously, but when the estimated arrival rate is higher than a predetermined level, some new calls are blocked irrespective of the availability of channels. Several CAC schemes based on interference and power control are proposed, where the acceptance of a new request depends on Signal-to-Interference Ratio (SIR) value $[2,6]$.
However, during the complexity of CAC in wireless environment, many simplified models and assumptions are made. Some schemes consider that each mobile node will make hand-over to neighboring cells with equal probability, which may be not accurate in general. For this reason, in [7], the authors proposed a mobility prediction scheme that is motivated by computational learning theory. The authors derive the mobility prediction scheme from data compression techniques that are both theoretically optimal and good in practice.

Use of intelligent methods based on Fuzzy Logic (FL), Neural Networks (NN) and Genetic Algorithms (GA) can prove to be efficient for traffic control in telecommunication networks $[8,9,10,11,12,13,14,15,16]$.

In this paper, in order to deal with $\mathrm{CAC}$ in wireless cellular networks, we propose a CAC scheme based on fuzzy logic [17]. Conventional CAC schemes for wireless networks must consider some measured parameters to make the decision. However, in wireless networks due to the user mobility and varying of channel condition the measurement obtained are not accurate. Also, it is very difficult to obtain the complete statistics of the input traffic. Therefore, the CAC decision must be made based on the uncertain or inaccurate information. The proposed fuzzy based system has 2 parts: fuzzy prediction scheme and fuzzy admission control scheme. The user movement is obtained by GPS and the fuzzy decision is based on the user speed, angel and distance from the Base Station (BS). We implement and evaluate the proposed system by simulation. We compare its performance with Shadow Cluster Concept (SCC) [18]. The proposed scheme can achieve a better prediction of the user behavior and a good admission decision compared with SCC.

The structure of this paper is as follows. In Section 2, we present the SCC. In Section 3, we introduce the proposed system. In Section 4, we discuss the simulation results. Finally, some conclusions are given in Section 5.

\section{Shadow Cluster Concept}

The wireless network must provide the requested level of service even if an active mobile terminal moves to a congested cell. In this case, the corresponding BS must provide the expected service even if this implies denying network access to new connection requests. Ideally, BSs should deny network access to certain connection requests only when it is strictly necessary. This constitutes a problem that could only be optimized if knowledge were available regarding the future movement and call holding times of the active mobile terminals in the wireless network, as well as the future movements and call holding times of the mobile terminals with connection requests. As in related problems, solutions close to optimal can be obtained by using knowledge of past events to predict future behavior [18]. 
The fundamental idea of the SCC is that every mobile terminal with an active wireless connection exerts an influence upon the cells (and their BSs) in the vicinity of its current location and along its direction of travel. As an active mobile terminal travels to other cells, the region of influence also moves, following the active mobile terminal to its new location. The BSs (and their cells) currently being influenced are said to form a shadow cluster, because the region of influence follows the movements of the active mobile terminal like a shadow, as shown in Fig. 1. The shadow (and therefore the level of influence) is strongest near the active mobile terminal, and fades away depending on factors such as the distance to the mobile terminal, current call holding time and priority, bandwidth resources being used, and the mobile terminal's trajectory and velocity. Because of these factors, the shape of a shadow cluster is usually not circular and can change over time. The center of a shadow cluster is not the geometric center of the area described by the shadow, but the cell where the mobile terminal is currently located. This cell is considered as the mobile terminal's current home cell. A bordering neighbor is a cell that shares a common border with the shadow cluster's center cell. In contrast, a non-bordering neighbor cell, although being a part of the shadow cluster, does not share a border with the shadow cluster's center cell.

Conceptually, the number and "darkness" of the shadows covering a cell reflect the amount of resources that the cell's BS needs to reserve in order to support the active mobile terminals currently in its own and in neighboring cells. With the information provided by shadow clusters, BSs can determine, for each new call request, whether the request can be supported by the wireless network. In practice, a shadow cluster is a virtual message system where BSs share probabilistic information with their neighbors on the likelihood that their active mobile terminals will move to neighbor cells (while remaining active) in the near future. With the information provided by shadow clusters, BSs project future demands and reserve resources accordingly. BSs reserve resources by denying network access to new call requests, and by "waiting" for active users to end their calls.

The decision process for the acceptance of a new call request also involves a shadow cluster. Every new call request results in the implementation of a tentative shadow cluster. BSs exchange information on their new call requests, and decide, based on this and other information, which requests should be accepted and which requests should be denied.

After a handoff, BSs within the old shadow cluster are notified about this movement, and the mobile terminal's new current BS has to assume the responsibility of supplying the appropriate information to the BSs within the new shadow cluster. BSs which were in an old shadow cluster that has just moved away must delete any entries corresponding to the active mobile terminal that established

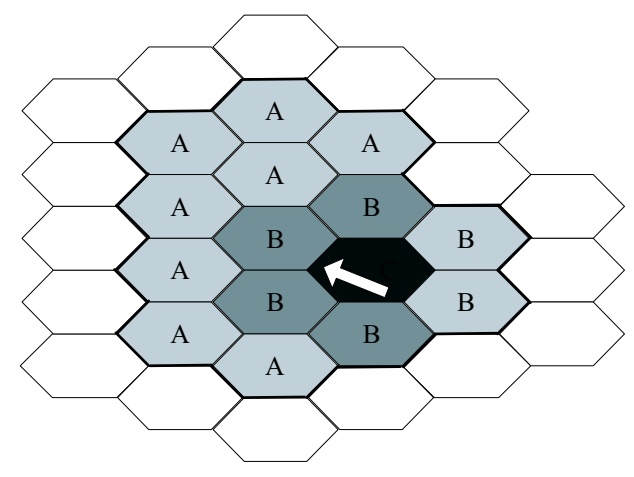

Figure 1. SCC.

the shadow cluster, and free reserved resources if appropriate. BSs which become part of the influence region of a shadow cluster must be given appropriate information on the shadow cluster's active mobile terminal, such as the respective QoS requirements, e.g., bandwidth demands, call dropping probabilities, and any other useful information such as the wireless connection's elapsed time, for the establishment of the new shadow cluster.

\section{Proposed Fuzzy Admission Control System}

The Fuzzy Logic Controller (FLC) is the main part of the proposed Fuzzy Admission Control System (FACS) and its basic elements are shown in Fig. 2. They are the fuzzifier, inference engine, Fuzzy Rule Base (FRB) and defuzzifier. As membership functions, we use triangular and trapezoidal membership functions because they are suitable for real-time operation [17]. They are shown in Fig. 3 and are given as:

$$
\begin{gathered}
f\left(x ; x_{0}, a_{0}, a_{1}\right)= \begin{cases}\frac{x-x_{0}}{a_{0}}+1 & \text { for } x_{0}-a_{0}<x \leq x_{0} \\
\frac{x_{0}-x}{a_{1}}+1 & \text { for } x_{0}<x \leq x_{0}+a_{1} \\
0 & \text { otherwise }\end{cases} \\
g\left(x ; x_{0}, x_{1}, a_{0}, a_{1}\right)= \begin{cases}\frac{x-x_{0}}{a_{0}}+1 & \text { for } x_{0}-a_{0}<x \leq x_{0} \\
1 & \text { for } x_{0}<x \leq x_{1} \\
\frac{x_{1}-x}{a_{1}}+1 & \text { for } x_{1}<x \leq x_{1}+a_{1} \\
0 & \text { otherwise }\end{cases}
\end{gathered}
$$

where $x_{0}$ in $f($.$) is the center of triangular function; x_{0}\left(x_{1}\right)$ in $g($.$) is the left (right) edge of trapezoidal function; and$ $a_{0}\left(a_{1}\right)$ is the left (right) width of the triangular or trapezoidal function.

The proposed FACS considers the following parameters for acceptance decision: user Speed $(S)$, user Angle $(A)$, Distance between user and BS $(D)$, Correction value $(C v)$, Required bandwidth $(R)$, Counter state $(C s)$, Accept or Reject decision $(A / R)$, Differentiated service $(D s)$, Real Time Counter $(R T C)$, and Non Real Time Counter (NRTC). The structure of the proposed FACS is shown in Fig. 4. 


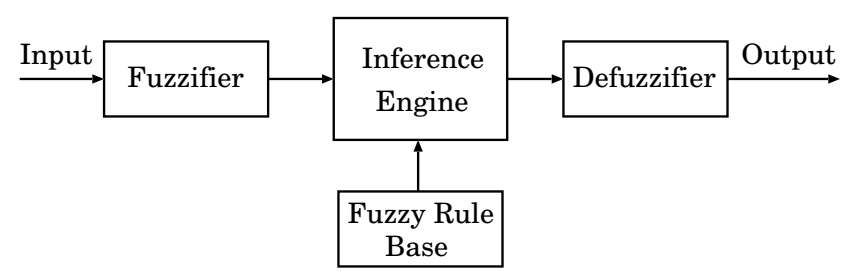

Figure 2. FLC structure.
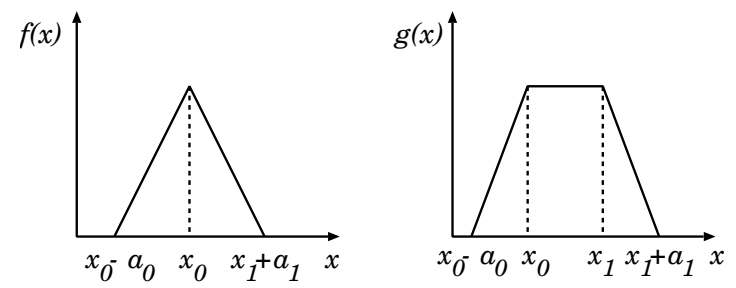

Figure 3. Triangular and trapezoidal membership functions.

\subsection{FLC1 Design}

The input parameters for FLC1 are: user Speed $(S)$, user Angle $(A)$, and the Distance between user and BS $(D)$, while the output linguistic parameter is Correction value $(C v)$. The term sets of $S, A$, and $D$ are defined respectively as:

$$
\begin{aligned}
T(S)= & \{\text { Slow, Middle, Fast }\}=\{\text { Sl, } M, F a\} \\
T(A)= & \{\text { Back } 1, \text { Left } 1, \text { Left } 2, \text { Straight, Right } 1, \text { Right } 2, \\
& \text { Back } 2\}=\{B 1, L 1, \text { L2,St,R } 1, \text { R2, B } 2\} \\
T(D)= & \{\text { Near, Far }\}=\{N, F\} .
\end{aligned}
$$

The membership functions for input parameters of FACS are defined as follows:

$$
\begin{aligned}
\mu_{S l}(S) & =g\left(S ; S l_{0}, S l_{1}, S_{w 0}, S_{w 1}\right) ; \\
\mu_{M}(S) & =f\left(S ; M_{0}, M_{w 0}, M_{w 1}\right) ; \\
\mu_{F a}(S) & =g\left(S ; F a_{0}, F a_{1}, F a_{w 0}, F a_{w 1}\right) ; \\
\mu_{B 1}(A) & =g\left(A ; B 1_{0}, B 1_{1}, B 1_{w 0}, B 1_{w 1}\right) ; \\
\mu_{L 1}(A) & =f\left(A ; L 1_{0}, L 1_{w 0}, L 1_{w 1}\right) ; \\
\mu_{L 2}(A) & =f\left(A ; L 2_{0}, L 2_{w 0}, L 2_{w 1}\right) ; \\
\mu_{S t}(A) & =f\left(A ; S t_{0}, S t_{w 0}, S t_{w 1}\right) ; \\
\mu_{R 1}(A) & =f\left(A ; R 1_{0}, R 1_{w 0}, R 1_{w 1}\right) ; \\
\mu_{R 2}(A) & =f\left(A ; R 2_{0}, R 2_{w 0}, R 2_{w 1}\right) ; \\
\mu_{B 2}(A) & =g\left(A ; B 2_{0}, B 2_{1}, B 2_{w 0}, B 2_{w 1}\right) ; \\
\mu_{N}(D) & =f\left(D ; N_{0}, N_{w 0}, N_{w 1}\right) ; \\
\mu_{F}(D) & =f\left(D ; F a_{0}, F a_{w 0}, F a_{w 1}\right) .
\end{aligned}
$$

The small letters $w 0$ and $w 1$ mean left width and right width, respectively.

The term set of the output linguistic parameter $T(C v)$ is defined as $\{$ Correction value 1, Correction value $2, \ldots$,

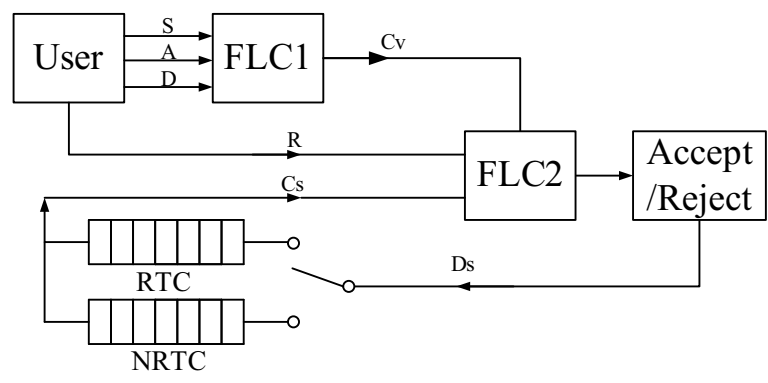

Figure 4. Proposed system model.

Correction value 9$\}=\{C v 1, C v 2, \ldots, C v 9\}$. The membership functions for the output parameter $C v$ are defined as follows:

$$
\begin{aligned}
& \mu_{C v 1}(C v)=g\left(C v ; C v 1_{0}, C v 1_{1}, C v 1_{w 0}, C v 1_{w 1}\right) \\
& \mu_{C v 2}(C v)=f\left(C v ; C v 2_{0}, C v 2_{w 0}, C v 2_{w 1}\right) ; \\
& \mu_{C v 3}(C v)=f\left(C v ; C v 3_{0}, C v 3_{w 0}, C v 3_{w 1}\right) \\
& \mu_{C v 4}(C v)=f\left(C v ; C v 4_{0}, C v 4_{w 0}, C v 4_{w 1}\right) \\
& \mu_{C v 5}(C v)=f\left(C v ; C v 5_{0}, C v 5_{w 0}, C v 5_{w 1}\right) \\
& \mu_{C v 6}(C v)=f\left(C v ; C v 6_{0}, C v 6_{w 0}, C v 6_{w 1}\right) \\
& \mu_{C v 7}(C v)=f\left(C v ; C v 7_{0}, C v 7_{w 0}, C v 7_{w 1}\right) ; \\
& \mu_{C v 8}(C v)=f\left(C v ; C v 8_{0}, C v 8_{w 0}, C v 8_{w 1}\right) \\
& \mu_{C v 9}(C v)=g\left(C v ; C v 9_{0}, C v 9_{1}, C v 9_{w 0}, C v 9_{w 1}\right) .
\end{aligned}
$$

The membership functions of FLC1 are shown in Fig. 5. The FRB forms a fuzzy set of dimensions $|T(S)| \times|T(A)| \times$ $|T(D)|$, where $|T(x)|$ is the number of terms on $T(x)$. The FRB1 shown in Table 1 has 42 rules. The control rules have the following form: IF "conditions" THEN "control action".

\subsection{FLC2 Design}

The input parameters for FLC2 are: the output parameter of the FLC1 $(C v)$, user Request $(R)$, and the Counter state $(C s)$, which shows the capacity of the system. While, the output linguistic parameter is the Accept/Reject decision $(A / R)$.

The term sets of $C v, R$, and $C s$ are defined as:

$$
\begin{aligned}
T(C v) & =\{\text { Bad, Normal, Good }\}=\{B, N, G\} \\
T(R) & =\{\text { Text }, \text { Voice, }, \text { ideo }\}=\{T, V o, V i\} \\
T(C s) & =\{\text { Small, Middle, Full }\}=\{S, M, F\} .
\end{aligned}
$$

In order to have a soft admission decision, for the output linguistic parameter $(A / R)$, we considered not only "accept" and "reject" but also "weak accept", "weak reject", and "not accept not reject" for the accept/reject decision. The membership functions for input and output linguistic parameters of FLC2 are shown in Fig. 6. The FRB2 shown in Table 2 has 27 rules. 


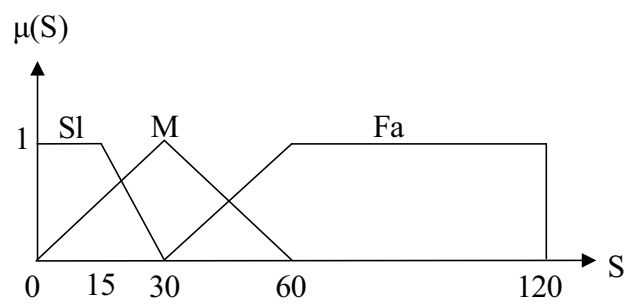

(a)

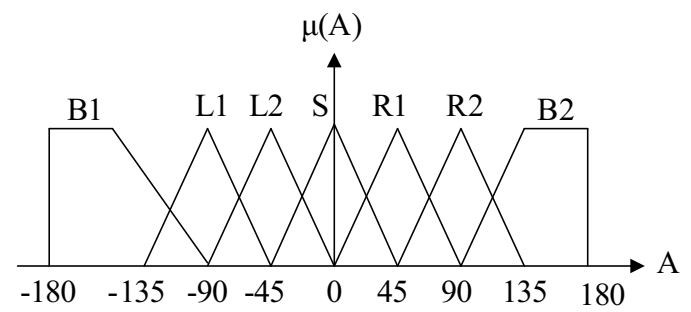

(b)

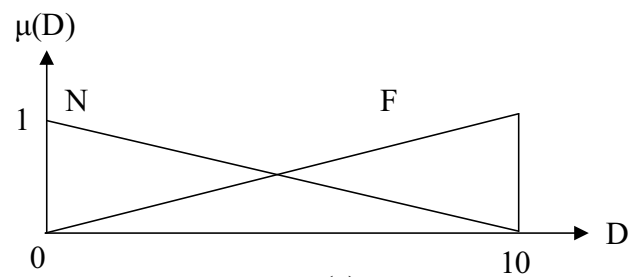

(c)

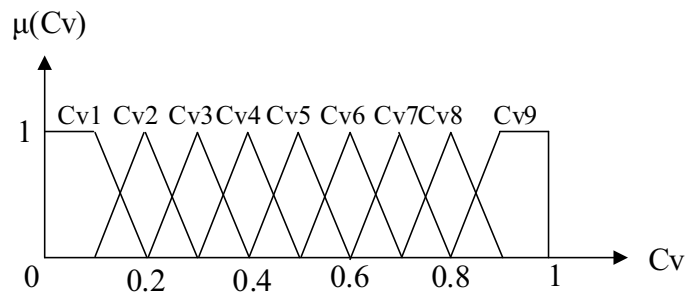

(d)

Figure 5. FLC1 membership functions.

The membership functions for input parameters of FLC2 are defined as follows:

$$
\begin{aligned}
\mu_{B}(C v) & =f\left(C v ; B_{0}, B_{w 0}, B_{w 1}\right) \\
\mu_{N}(C v) & =f\left(C v ; N_{0}, N_{w 0}, N_{w 1}\right) ; \\
\mu_{G}(C v) & =f\left(C v, G_{0}, G_{w 0}, G_{w 1}\right) \\
\mu_{T}(R) & =f\left(R ; T_{0}, T_{w 0}, T_{w 1}\right) \\
\mu_{V o}(R) & =f\left(R ; V o_{0}, V o_{w 0}, V o_{w 1}\right) ; \\
\mu_{V i}(R) & =f\left(R ; V i_{0}, V i_{w 0}, V i_{w 1}\right) ; \\
\mu_{S}(C s) & =f\left(C s ; S_{0}, S_{w 0}, S_{w 1}\right) ; \\
\mu_{M}(C s) & =f\left(C s ; M_{0}, M_{w 0}, M_{w 1}\right) \\
\mu_{F}(C s) & =f\left(C s ; F_{0}, F_{w 0}, F_{w 1}\right) ;
\end{aligned}
$$

\begin{tabular}{|c|c|c|c|c|}
\hline Rule & $S$ & A & D & $\mathrm{Cv}$ \\
\hline 0 & S1 & B1 & $\mathrm{N}$ & Cv3 \\
\hline 1 & Sl & B1 & $\mathrm{F}$ & Cv1 \\
\hline 2 & S1 & L1 & $\mathrm{N}$ & $\mathrm{Cv} 4$ \\
\hline 3 & S1 & L1 & $\mathrm{F}$ & $\mathrm{Cv} 2$ \\
\hline 4 & $\mathrm{Sl}$ & L2 & $\mathrm{N}$ & $\mathrm{Cv} 5$ \\
\hline 5 & S1 & L2 & $\mathrm{F}$ & Cv3 \\
\hline 6 & S1 & St & $\mathrm{N}$ & $\mathrm{Cv} 9$ \\
\hline 7 & Sl & $\mathrm{St}$ & $\mathrm{F}$ & Cv3 \\
\hline 8 & S1 & R1 & $\mathrm{N}$ & Cv5 \\
\hline 9 & Sl & $\mathrm{R} 1$ & $\mathrm{~F}$ & $\mathrm{Cv} 2$ \\
\hline 10 & S1 & $\mathrm{R} 2$ & $\mathrm{~N}$ & $\mathrm{Cv} 4$ \\
\hline 11 & $\mathrm{~S} 1$ & $\mathrm{R} 2$ & $\mathrm{~F}$ & $\mathrm{Cv} 2$ \\
\hline 12 & $\mathrm{~S} 1$ & B2 & $\mathrm{N}$ & Cv3 \\
\hline 13 & S1 & B2 & $\mathrm{F}$ & $\mathrm{Cv} 1$ \\
\hline 14 & M & B1 & $\mathrm{N}$ & $\mathrm{Cv} 2$ \\
\hline 15 & M & B1 & $\mathrm{F}$ & $\mathrm{Cv} 1$ \\
\hline 16 & $\mathrm{M}$ & L1 & $\mathrm{N}$ & $\mathrm{Cv} 4$ \\
\hline 17 & M & L1 & $\mathrm{F}$ & $\mathrm{Cv} 1$ \\
\hline 18 & M & L2 & $\mathrm{N}$ & $\mathrm{Cv} 8$ \\
\hline 19 & M & L2 & $\mathrm{F}$ & Cv5 \\
\hline 20 & M & St & $\mathrm{N}$ & $\mathrm{Cv} 9$ \\
\hline 21 & M & St & $\mathrm{F}$ & $\mathrm{Cv} 7$ \\
\hline 22 & $\mathrm{M}$ & R1 & $\mathrm{N}$ & Cv8 \\
\hline 23 & M & $\mathrm{R} 1$ & $\mathrm{~F}$ & Cv5 \\
\hline 24 & M & $\mathrm{R} 2$ & $\mathrm{~N}$ & $\mathrm{Cv} 4$ \\
\hline 25 & $\mathrm{M}$ & $\mathrm{R} 2$ & $\mathrm{~F}$ & Cv1 \\
\hline 26 & M & B2 & $\mathrm{N}$ & $\mathrm{Cv} 2$ \\
\hline 27 & M & B2 & $\mathrm{F}$ & Cv1 \\
\hline 28 & $\mathrm{Fa}$ & B1 & $\mathrm{N}$ & Cv1 \\
\hline 29 & $\mathrm{Fa}$ & B1 & $\mathrm{F}$ & Cv1 \\
\hline 30 & $\mathrm{Fa}$ & L1 & $\mathrm{N}$ & Cv1 \\
\hline 31 & $\mathrm{Fa}$ & L1 & $\mathrm{F}$ & $\mathrm{Cv} 2$ \\
\hline 32 & $\mathrm{Fa}$ & L2 & $\mathrm{N}$ & Cv6 \\
\hline 33 & $\mathrm{Fa}$ & L2 & $\mathrm{F}$ & Cv8 \\
\hline 34 & $\mathrm{Fa}$ & $\mathrm{St}$ & $\mathrm{N}$ & $\mathrm{Cv} 9$ \\
\hline 35 & $\mathrm{Fa}$ & St & $\mathrm{F}$ & $\mathrm{Cv} 9$ \\
\hline 36 & $\mathrm{Fa}$ & $\mathrm{R} 1$ & $\mathrm{~N}$ & Cv6 \\
\hline 37 & $\mathrm{Fa}$ & R1 & $\mathrm{F}$ & Cv8 \\
\hline 38 & $\mathrm{Fa}$ & $\mathrm{R} 2$ & $\mathrm{~N}$ & $\mathrm{Cv} 1$ \\
\hline 39 & $\mathrm{Fa}$ & $\mathrm{R} 2$ & $\mathrm{~F}$ & $\mathrm{Cv} 2$ \\
\hline 40 & $\mathrm{Fa}$ & B2 & $\mathrm{N}$ & Cv1 \\
\hline 41 & $\mathrm{Fa}$ & B2 & $\mathrm{F}$ & Cv1 \\
\hline
\end{tabular}

The term set of the output linguistic parameter $T(A / R)$ is defined as $\{$ Reject, Weak Reject, Not Reject Not Accept, Weak Accept, Accept $\}$. We write for short as $\{\mathrm{R}, \mathrm{WR}$, NRNA, WA, A $\}$. The membership functions for the output parameter $A / R$ are defined as follows:
Table 1. FRB1.

$$
\begin{aligned}
\mu_{R}(A / R) & =g\left(A / R ; R_{0}, R_{1}, R_{w 0}, R_{w 1}\right) ; \\
\mu_{W R}(A / R) & =f\left(A / R ; W R_{0}, W R_{w 0}, W R_{w 1}\right) ; \\
\mu_{N R N A}(A / R) & =f\left(A / R ; N R N A_{0}, N R N A_{w 0}, N R N A_{w 1}\right) ; \\
\mu_{W A}(A / R) & =f\left(A / R ; W A_{0}, W A_{w 0}, W A_{w 1}\right) ; \\
\mu_{A}(A / R) & =g\left(A / R ; A_{0}, A_{1}, A_{w 0}, A_{w 1}\right) .
\end{aligned}
$$

\section{Simulation Results}

The simulation were carried out in Linux Fedora Core5 computer. We considered the following parameters for simulations: the user speed was from 0 to $120 \mathrm{~km} / \mathrm{h}$, the user direction was changed from -180 degree to +180 degree, the 


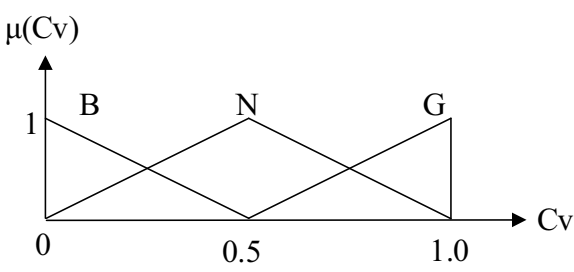

(a)

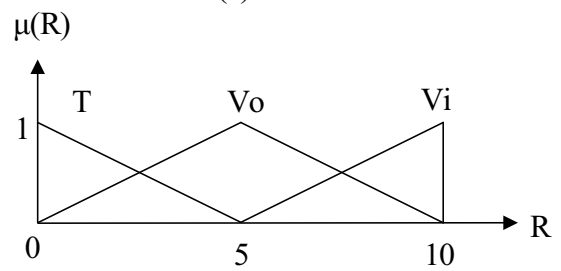

(b)

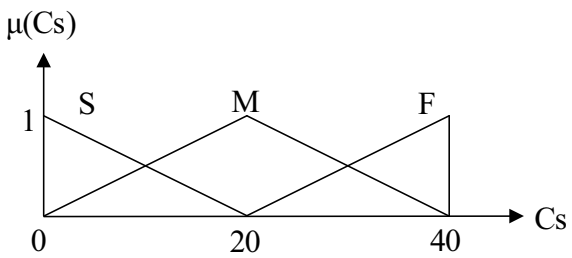

(c)

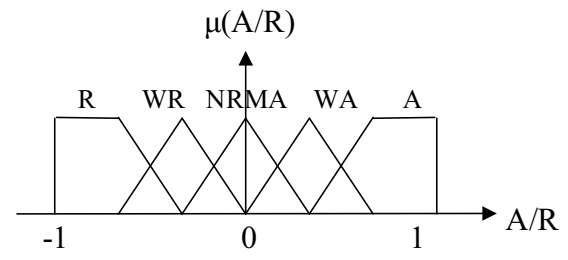

(d)

Figure 6. FLC2 membership functions.

distance between users and BS was changed between 0 to $10 \mathrm{~km}$. The required bandwidth for voice, video and text was $30 \%, 10 \%$, and $60 \%$, respectively. The requested size was 1, 5 and 10 Bandwidth Units (BU) for text, voice and video, respectively. The bandwidth of the BS was considered 40 BU.

In Fig. 7 is shown the relation between percentage of accepted calls versus number of requesting connections. In this simulation, we consider the user speed as a parameter. From the simulation results can be seen that with the increase of the user speed, the percentage of the number of the accepted calls is increased when the number of requesting connections increases. This happens because with the increase of the user speed, the user direction can not be changed easy, this results in a better prediction of the user direction and the network resources are used better. On the other hand, when the user speed is $4 \mathrm{~km} / \mathrm{h}$ or $10 \mathrm{~km} / \mathrm{h}$, the user direction can be changed (this is the case for walking users). For this reason, the prediction of the user direction becomes difficult, which results in a small percentage of the accepted calls.
Table 2. FRB2.

\begin{tabular}{|l|l|l|l|l|}
\hline \hline Rule & CV & R & Cs & A/R \\
\hline 0 & B & T & S & A \\
1 & B & T & M & NRNA \\
2 & B & T & F & NRNA \\
3 & B & Vo & S & A \\
4 & B & Vo & M & NRNA \\
5 & B & Vo & F & WR \\
6 & B & Vi & S & WA \\
7 & B & Vi & M & NRNA \\
8 & B & Vi & F & WR \\
9 & N & T & S & A \\
10 & N & T & M & NRNA \\
11 & N & T & F & NRNA \\
12 & N & Vo & S & A \\
13 & N & Vo & M & NRNA \\
14 & N & Vo & F & NRNA \\
15 & N & Vi & S & WA \\
16 & N & Vi & M & NRNA \\
17 & N & Vi & F & NRNA \\
18 & G & T & S & A \\
19 & G & T & M & A \\
20 & G & T & F & NRNA \\
21 & G & Vo & S & A \\
22 & G & Vo & M & A \\
23 & G & Vo & F & WR \\
24 & G & Vi & S & A \\
25 & G & Vi & M & A \\
26 & G & Vi & F & R \\
\hline
\end{tabular}

In Fig. 8, we consider the angle as parameter. We show the simulation results for different angels from 0 to $90 \mathrm{de}-$ gree. When the user angle is not changed, the percentage of accepted calls is close to $100 \%$ and is decreased with the increase of the number of requesting connections. With the increase of the angle, the user is going far from the BS, so there is not need to allocate the bandwidth for this user. This is why the percentage of the number of accepted calls is decreased with the increase of the angle value. When the angle is more than 90 degree, the percentage of accepted calls is almost zero. For this reason, we did not show in this figure.

In Fig. 9, the distance between the user and BS is considered as a parameter. With the increase of the number of requesting connections, the percentage of accepted calls is decreased. Also, when the distance between the user and $\mathrm{BS}$ is increased, the percentage of the accepted calls is decreased. However, the difference is not so big like in the case of the speed and angle. This shows that the speed and angle have strong effect compared with the distance.

In order to evaluate the performance of the proposed system, we compare its performance with SCC. The simulation results are shown in Fig. 10. When the number of requesting connections is less than 50 , the percentage of accepted calls for proposed system is higher than SCC. However, when the number of requesting connections is larger than 50, the proposed system accepts less number of con- 


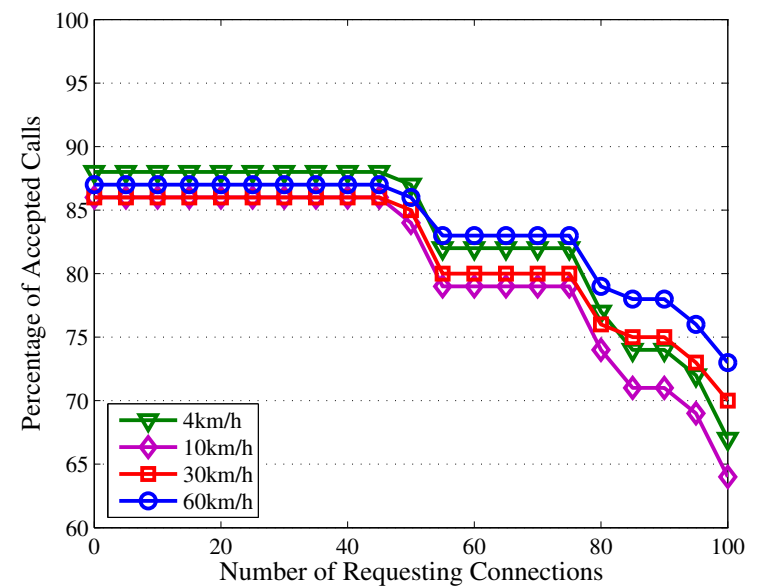

Figure 7. Percentage of number of accepted calls vs. number of requesting connections for different speed values.

nections. This is because, the proposed system guarantees the QoS of ongoing calls and also considers the QoS for the requesting connections. When the number of requesting connections is less than 50 (the case when there is enough $\mathrm{BU})$, the proposed system make a better allocation of the resources compared with SCC.

\section{Conclusions}

In this paper, we proposed a fuzzy based admission control system (called FACS) for wireless cellular networks. We evaluated the performance of the proposed system for different scenarios. We also compared the performance of the proposed system with conventional SCC. From the simulations results, we conclude:

- with the increase of the user speed, the percentage of the number of the accepted calls is increased when the number of requesting connections increases;

- when the user speed is slow (the case for walking users), the prediction of the user direction becomes difficult, because the users can change their direction, which results in a small percentage of the accepted calls;

- when the user angle is not changed, the percentage of accepted calls is close to $100 \%$ and is decreased with the increase of the number of requesting connections;

- when the distance between the user and BS is increased, the percentage of the accepted calls is decreased, but the difference is not so big like in the case of the speed and angle;

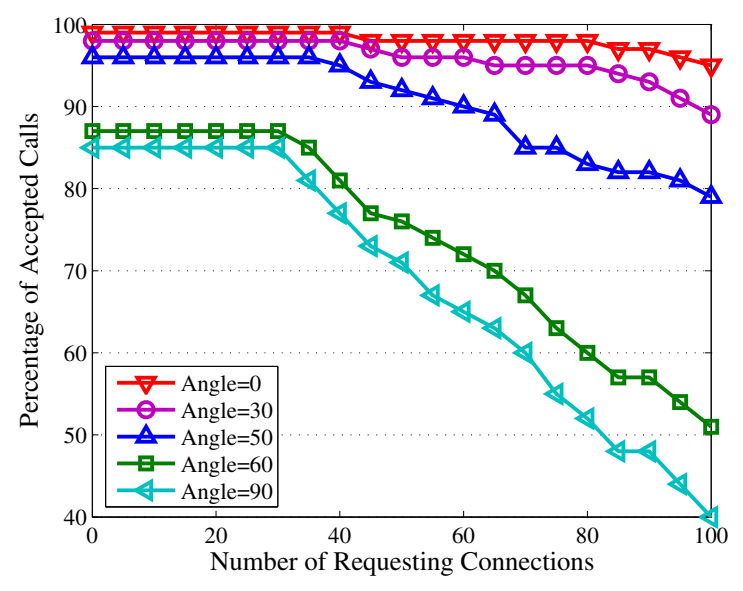

Figure 8. Percentage of number of accepted calls vs. number of requesting connections for different angle values.

- the proposed system has better prediction and acceptance decision than SCC.

In this work, we did not consider the priority of the ongoing calls and requesting connections. This will be a problem to treated in the future work.

\section{References}

[1] Y. Fang, and Y. Zhang, "Call Admission Control Schemes and Performance Analysis in Wireless Mobile Networks", IEEE Trans. on Vehicular Technology, Vol. 51, No. 2, pp. 371-382, March 2002.

[2] W. Wang, X. Wang, and A. A. Nilsson, "EnergyEfficient Bandwidth Allocation in Wireless Networks: Algorithms, Analysis, and Simulations", IEEE Transactions on Wireless Communications, Vol 5, No. 5, pp. 1103-1114, May 2006.

[3] D. Z. Deniz and N. O. Mohamed, "Performance of CAC Strategies for Multimedia Traffic in Wireless Networks", IEEE Journal on Selected Areas in Commun., Vol. 21, No. 10, pp. 1557-1565, December 2003.

[4] N. Bartolini, and I. Chamtac, "Call Admission Control in Wireless Multimedia Networks", Proc. of the 13th IEEE International Symposium on Personal Indoor Mobile Radio Communication (PIMRC-2002), Vol. 1, pp. 285-289, September 2002.

[5] S. Kovvuri, V. Pandey, D. Ghosal, B. Mukherjee, D. Sarkar, "A Call-Admission Control (CAC) Algorithm for Providing Guaranteed QoS in Cellular Net- 


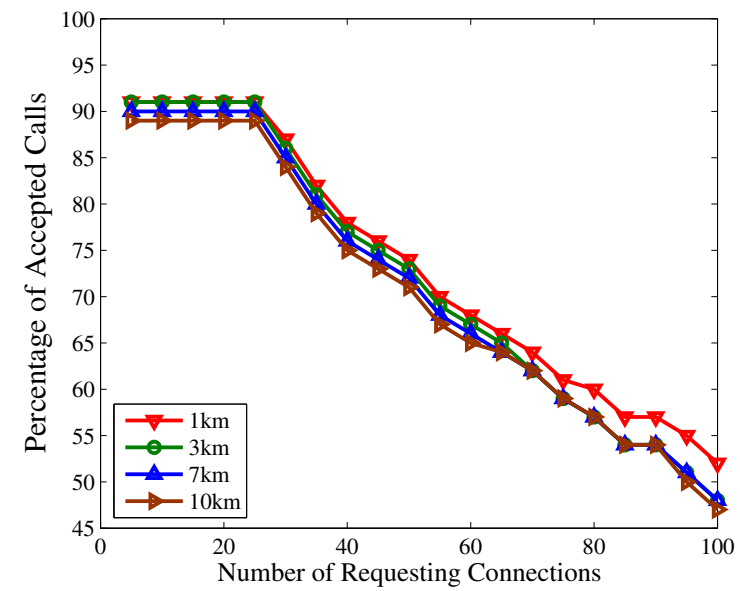

Figure 9. Percentage of number of accepted calls vs. number of requesting connections for different distance values.

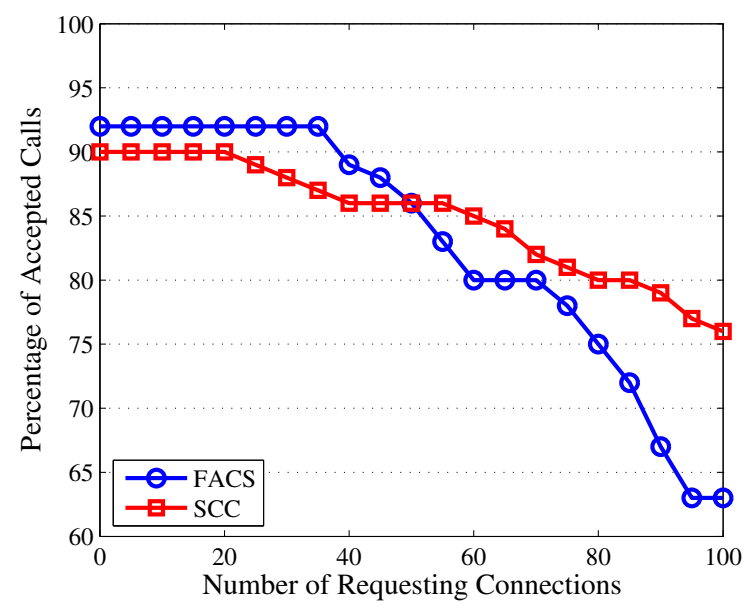

Figure 10. Performance of proposed system and SCC.

works", International Journal of Wireless Information Networks, Vol. 10, No. 2, pp. 73-85, April 2003.

[6] M. Xiao, N. B. Shroff, E. K. P. Chong, "Distributed Admission Control for Power-Controlled Cellular Wireless Systems", IEEE/ACM Trans. on Networking, Vol. 9, No. 6, pp. 790-800, December 2001.

[7] F. Yu and V. C. M. Leung, "Mobility-Based Predictive Call Admission Control and Bandwidth Reservation in Wireless Cellular Networks", Proc. of IEEE INFOCOM-2001, pp. 518-526, 2001.

[8] L. Barolli, A. Koyama, T. Yamada, S. Yokoyama, T. Suganuma, N.Shiratori, "A Fuzzy Admission Control Scheme and Its Performance Evaluation", IPSJ Journal, Vol. 42, No. 12, pp. 3213-3221, December 2001.
[9] L. Barolli, A. Koyama, T. Suganuma and N. Shiratori, "A Genetic Algorithm Based QoS Routing Method for Multimedia Communications Over High-Speed Networks", IPSJ Journal, Vol. 44, No. 2, pp. 544-552, February 2003.

[10] B. Bensaou, S. T. C. Lam, H-W. Chu, D. H. K. Tsang, "Estimation of the Cell Loss Ratio in ATM Networks with a Fuzzy System and Application to Measurement-Based Call Admission Control", IEEE/ACM Trans. on Networking, Vol. 5, No. 4, pp. 572-584, August 1997.

[11] I. Habib, (Ed.), "Neurocomputing in High-Speed Networks", IEEE Commun. Magazine, Special Issue, Vol. 33, No. 10, 1995.

[12] P. M. L. Chan, R.E. Sheriff, Y.F. Hu, P. Conforto, C. Tocci, "Mobility Management Incorporating Fuzzy Logic for a Heterogeneous IP Environment", IEEE Communications Magazine, Vol. 39, No. 12, pp. 4251, December 2001.

[13] W. Zhang, "Handover Decision Using Fuzzy MADM in Heterogeneous Networks", IEEE Wireless Communications and Networking Conference (WCNC-2004), Vol. 2, pp. 653-658, 2004.

[14] T. Onel, C. Ersoy, E. Cayirci, ”A Fuzzy Inference System for the Handoff Decision Algorithms in the Virtual Cell Layout Base Tactical Communications System”, IEEE Military Communications Conference (MILCO-2002), Vol.1, pp. 436-441, October 2002.

[15] L. Barolli, A. Koyama, T. Suganuma, N. Shiratori, "GAMAN: A GA Based QoS Routing Method for Mobile Ad-hoc Networks", Journal of Interconnection Networks (JOIN), Vol. 4, No. 3, pp. 251-270, September 2003 .

[16] Q. Liang, "A Design Methodology for Wireless Personal Area Networks with Power Efficiency", IEEE Wireless Communications and Networking Conference (WCNC-2003), Vol. 3, pp. 1475-1480, 2003.

[17] D. Dubois, H. Prade, R. Yager, (Eds.), "Fuzzy Sets for Intelligent Systems", Morgan Kaufman Publishers, 1993.

[18] D. A. Levine, I. F. Akyildiz, M. Naghshineh, ”A Resource Estimation and Call Admission Algorithm for Shadow Cluster Concept", IEEE/ACM Transactions on Networking, Vol. 5, No. 1, pp. 1-12, 1997. 